\title{
Signaling Pathway Activities Improve Prognosis for Breast Cancer
}

Yunlong Jiao ${ }^{1,2,3,4}$, Marta R. Hidalgo ${ }^{5}$, Cankut Çubuk ${ }^{6}$, Alicia Amadoz ${ }^{5}$, José CarbonellCaballero $^{5}$, Jean-Philippe Vert ${ }^{1,2,3,4}$, and Joaquín Dopazo ${ }^{6,7,8, *}$

${ }^{1}$ MINES ParisTech, PSL Research University, Centre for Computational Biology, 77300 Fontainebleau, France; ${ }^{2}$ Institut Curie, 75248 Paris Cedex, Franc; ${ }^{3}$ INSERM, U900, 75248 Paris Cedex, France; ${ }^{4}$ Ecole Normale Supérieure, Department of Mathematics and their Applications, 75005 Paris, France; ${ }^{5}$ Computational Genomics Department, Centro de Investigación Príncipe Felipe (CIPF), 46012 Valencia, Spain; ${ }^{6}$ Clinical Bioinformatics Research Area, Fundación Progreso y Salud (FPS), Hospital Virgen del Rocío, 41013, Sevilla, Spain; ${ }^{7}$ Functional Genomics Node (INB), FPS, Hospital Virgen del Rocío, 41013 Sevilla, Spain; ${ }^{8}$ Bioinformatics in Rare Diseases (BiER), Centro de Investigación Biomédica en Red de Enfermedades Raras (CIBERER), FPS, Hospital Virgen del Rocío, 41013, Sevilla, Spain

*To whom correspondence should be addressed.

\begin{abstract}
With the advent of high-throughput technologies for genome-wide expression profiling, a large number of methods have been proposed to discover gene-based signatures as biomarkers to guide cancer prognosis. However, it is often difficult to interpret the list of genes in a prognostic signature regarding the underlying biological processes responsible for disease progression or therapeutic response. A particularly interesting alternative to gene-based biomarkers is mechanistic biomarkers, derived from signaling pathway activities, which are known to play a key role in cancer progression and thus provide more informative insights into cellular functions involved in cancer mechanism. In this study, we demonstrate that pathway-level features, such as the activity of signaling circuits, outperform conventional gene-level features in prediction performance in breast cancer prognosis. We also show that the proposed classification scheme can even suggest, in addition to relevant signaling circuits related to disease outcome, a list of genes that do not code for signaling proteins whose contribution to cancer prognosis potentially supplements the mechanisms detected by pathway analysis.
\end{abstract}

\section{Introduction}

Over the past decades, many efforts have been addressed to the identification of gene-based signatures to predict patient prognosis using gene expression data (Paik, et al., 2004; Sotiriou and Pusztai, 2009; van 't Veer, et al., 2002; Wang, et al., 2005). Despite the success of its use, gene expression signatures have not been exempt of problems (Ein-Dor, et al., 2006; Iwamoto and Pusztai, 2010). Specifically, one major drawback of multi-gene biomarkers is that they often lack proper interpretation in terms of mechanistic link to the fundamental cell processes responsible for disease progression or therapeutic response (Dopazo, 2010; van't Veer and Bernards, 2008). Actually, it is increasingly recognized that complex traits, such as disease or drug response, are better understood as alterations in the operation of functional modules caused by different combinations of gene perturbations (Barabasi, et al., 2011; Barabasi and Oltvai, 2004; Oti and Brunner, 2007). To address this inherent complexity different methodologies have tried to exploit several functional module conceptual representations, such as protein interaction networks or pathways, to interpret gene expression data within a systems biology context (Barabasi, et al., 2011; Fryburg, et al., 2014; Hood, 2013; Vidal, et al., 2011). Actually, it has recently been shown that the pathway-level representation generates clinically relevant stratifications and outcome predictors for glioblastoma and colorectal cancer (Drier, et al., 2013) and also breast cancer (Livshits, et al., 2015). Moreover, mathematical models of the activity of a pathway have demonstrated a significantly better association to poor prognosis in neuroblastoma patients than the activity of their constituent genes, including $M I C N$, a conventional biomarker (Fey, et al., 2015). This observation has recently been extended to other cancers (Hidalgo, et al., 2017) and to the prediction of drug effects (Amadoz, et al., 2015).

Given that the inferred activity of the pathway should be closely related to its cellular mechanism for disease progression, its use to guide cancer prognosis seems promising. Recently, a number of pathway activity inference methods have been proposed (Hidalgo, et al., 2017; Jacob, et al., 2012; Li, et al., 2015; Martini, et al., 2013). Here, we use the canonical circuit activity analysis method, which has demonstrated to have a superior performance (Hidalgo, et al., 2017) finding significant associations of specific circuit activities, 
bioRxiv preprint doi: https://doi.org/10.1101/132357; this version posted April 29, 2017. The copyright holder for this preprint (which was not certified by peer review) is the author/funder, who has granted bioRxiv a license to display the preprint in perpetuity. It is made available under aCC-BY 4.0 International license.

directly responsible for triggering the prominent cancer hallmarks (Hanahan and Weinberg, 2011), to patient survival. This method recodes gene expression values into measurements of signaling circuit activities that ultimately account for cell responses to specific stimuli. Such activity values can be considered multigenic mechanistic biomarkers that can be used as features for cancer prognosis.

We demonstrate that the activity of signaling circuits yields comparable or even better prediction in breast cancer prognosis than the expression of individual genes, while detected mechanistic biomarkers enjoy the compelling advantage of readily available interpretation in terms of the corresponding cellular functions they trigger. Moreover, we show that the proposed prediction scheme can even suggest, in addition to interesting signaling circuits related to disease outcome, a list of prognostic genes that do not code for signaling proteins whose contribution to cancer prognosis potentially supplements the mechanism included in the pathways modeled.

Table 1. Summary of survival outcome of the breast cancer patients in the TCGA dataset.

\begin{tabular}{llll}
\hline Donor vital status & Pseudo label & No. of & Percentage \\
samples & $14.1 \%$ \\
\hline Deceased (poor prognosis) & Positive & 124 & $85.9 \%$ \\
Alive (good prognosis) & Negative & 755 & $100.0 \%$ \\
Total & & 879 & \\
\hline
\end{tabular}

\section{Methods}

\section{Data source and processing}

The breast cancer gene expression and survival data used here was downloaded from The Cancer Genome Atlas (TCGA), release No. 20 of the International Cancer Genome Consortium (ICGC) data portal (https://dcc.icgc.org/releases/release_20/Projects/BRCA-US). This dataset provides the RNA-seq counts of 18,708 genes for 879 tumor samples, in which we also have records of the vital status of corresponding donors, namely the overall survival outcome of the cancer patients being alive or deceased at the end of clinical treatment (Table 1). Since TCGA cancer data are from different origins and underwent different management processes, non-biological experimental variations, commonly known as batch effect, associated to Genome Characterization Center (GCC) and plate ID must be removed from the RNA-seq data. The COMBAT method (Johnson, et al., 2007) was used for this purpose. We then applied the trimmed mean of M-values normalization method (TMM) method (Robinson and Oshlack, 2010) for data normalization which is essential in applying the CCAA method. The resulting normalized values were finally entered to the pathway analysis method.

A total of 60 KEGG pathways (Supplementary Table 1) were downloaded from the KEGG repository (Kanehisa, et al., 2012), including 2,212 gene products that participate in 3,379 nodes.

\section{Modeling framework for signaling pathways}

We applied the canonical circuit activity analysis method (Hidalgo, et al., 2017), as implemented in the hipathia $\mathrm{R}$ package available at https://github.com/babelomics/hipathia, in pursuit of modeling signaling activity. Within the modeling context, a circuit is defined as all possible routes the signal can traverse to be transmitted from a particular input node to a particular output node (see Supplementary Figure 1A). A total of 6,101 circuits are identified and modeled in this study. The transmission of the signal depends on the integrity of the chain of nodes that connect the receptor to the effector and briefly, it is estimated as follows. The presence of the mRNA (the normalized RNA-seq counts rescaled between 0 and 1) is taken as a proxy for the presence of the corresponding protein in each pathway node (Bhardwaj and Lu, 2005; Efroni, et al., 2007; Montaner, et al., 2009; Sebastian-Leon, et al., 2014). Then, the degree of integrity of the circuit is estimated by modeling the signal flow across it. Specifically, the input node (receptor) is initialized by an incoming signal of intensity value of 1 , and then for each node $n$ of the circuit, the signal value $s_{n}$ is updated by the following rule:

$$
S_{n}=v_{n} \cdot\left(1-\prod_{s_{a} \in A_{n}}\left(1-s_{a}\right)\right) \cdot \prod_{s_{i} \in I_{n}}\left(1-s_{i}\right)
$$

where $A n$ denotes the set of signals arriving to the node from activation edges, In denotes the set of signals arriving to the node from inhibition edges, and $v n$ is the (normalized) value of the current node $n$. 
bioRxiv preprint doi: https://doi.org/10.1101/132357; this version posted April 29, 2017. The copyright holder for this preprint (which was not certified by peer review) is the author/funder, who has granted bioRxiv a license to display the preprint in perpetuity. It is made available under aCC-BY 4.0 International license.

Finally, the activity value for the circuit is defined by the signal intensity transmitted through the last (effector) protein of the circuit which quantifies the cell function ultimately activated by the circuit.

Since output nodes at the end of circuits are the ultimate triggers of specific cellular actions, an effector circuit is defined from a functional viewpoint as a higher-level signaling entity that composes all circuits ending at the same output node. When applied to an effector circuit, the method returns the joint intensity of the signal arriving to the corresponding effector node (see Supplementary Figure 1B). Furthermore, the known functions triggered by each effector protein in cell can be derived from their functional annotations. Here we use UniProt (UniProt_Consortium, 2015) and Gene Ontology (Ashburner, et al., 2000) (GO) annotations.

Finally, inferred signaling activity values of those effector circuits ending at proteins with the same annotated functions are averaged to quantify the activity of the function realized in cell. This way we obtain estimated activity values directly connected to a list of cellular functions (Supplementary Figure 1C).

Supplementary Figure 1 depicts the different levels of abstraction from circuits, to effector circuits and finally functions. Eventually, a subset of curated functions can be used for a specific scenario in which the relevant functions are known. Here we use cancer hallmarks (Hanahan and Weinberg, 2011) .

\section{Cancer prognosis with inferred signaling pathway activity}

In this study, we are interested in evaluating the prognostic power of pathway-level mechanistic features and gene-based features alone and in combination. Using the hipathia method we recoded the list of gene expression values of each tumor sample into the corresponding lists of signaling activity values for the three levels of abstraction: circuits, effector circuits and functions, as described in UniProt and GO annotations. Therefore for each tumor sample we end up with a profile of gene expression, a profile of circuit signaling activity, a profile of effector circuit signaling activity, a profile of UniProt-based cellular function activity and a profile of GO-based cellular function activity. These profiles are sample-specific profiles that can be straightforwardly used as prognostic features using any classification algorithm. Note that pathway-level profiles are derived with no regard to any information provided by the genes whose products do not participate in cell signaling, and the prognostic power of pathway-level profiles may thus be limited by the coverage of genes in known biological pathways. In order to understand the relative contribution to the pathway-level profiles and gene-level profiles to the accurate separation between good vs poor prognosis, we devised four artificial profiles: path-gene expression profile containing only genes that are involved in the KEGG signaling pathways, other-gene expression profile containing only genes that are absent from the KEGG pathways, a combined profile consisting of signaling activity of effector circuits and expression of other-genes, and a combined profile consisting of signaling activity of circuits and expression of other-genes. Thus, we use a total of 9 types of profiles (detailed in Table 2) From the viewpoint of machine learning, this study is formulated as a typical binary classification problem where we determine a positive or negative pseudo label for each sample. Based on the data available in this study (Table 1) we perform a 5-fold cross-validation repeated 10 times on the dataset and report the mean performance over the $5 \times 10=50$ splits to assess the prognostic power for each type of profile. The performance is evaluated by the Area Under the ROC Curve (AUROC) criteria (Sing, et al., 2005). Note that usually a classifier returns a continuous prediction between 0 and 1 for each sample denoting the probability of that sample being in the positive class rather than in the negative class, and then assigns either label to the sample according to some cutoff value thresholding the prediction. In fact, AUROC is a cutoff-free score that measures the probability that the classifier will score a randomly drawn positive sample higher than a randomly drawn negative sample.

Table 2. Summary of different types of profiles used as predictive features for breast cancer prognosis in this study.

\begin{tabular}{llll}
\hline Alias & Profile type & Number features & Analysis level \\
\hline fun.vals & & 81 & Circuit- \\
go.vals & UniProt-based functions & 370 & Circuit- \\
eff.vals & GO-based functions & 1,038 & Circuit- \\
path.vals & Effector circuits & 6,101 & Circuit- \\
path.genes.vals & Circuits & 2,212 & Gene \\
other.genes.vals & Pathway-genes & 16,496 & Gene \\
genes.vals & Other-genes & Gll genes & 18,708
\end{tabular}


bioRxiv preprint doi: https://doi.org/10.1101/132357; this version posted April 29, 2017. The copyright holder for this preprint (which was not certified by peer review) is the author/funder, who has granted bioRxiv a license to display the preprint in perpetuity. It is made available under aCC-BY 4.0 International license.

eff.and.other.- genes.vals

path.and.other.- genes.vals
Effector and other-genes

Circuits and other-genes
17,534

22,597
Circuit + Gene

Circuit + Gene

In this study we consider 12 classification algorithms as candidate classifiers, most of which are state-ofthe-art (Table 3). When we assess the prognosis performance for a specific type of profile on a specific (external) cross-validation split of the data, we perform an internal 5-fold cross-validation on the training set to determine which classifier returns the highest cross-validated performance and the best classifier is then used on the test set to obtain the performance score. This procedure guarantees that the performance on each (external) cross-validation split is evaluated impartially for each profile with its best suited algorithm.

Table 3. The 12 classifiers considered in this study to classify prognosis for breast tumor samples. Note that majority voting classifier serves as a baseline negative-control model which outputs a constant label for any test sample by the dominant class in the training set..

\begin{tabular}{|c|c|c|}
\hline Alias & Classifier & Reference \\
\hline LinearSVM & Support Vector Machine with linear kernel & (Chang and Lin, 2011) \\
\hline KendallSVM & Support Vector Machine with Kendall kernel & (Jiao and Vert, 2016; Zeileis, et al., 2004) \\
\hline KNN & k-nearest neighbor classifier & (Ripley, 2007; Venables and Ripley, 2002) \\
\hline SparseSVM & L1-regularized L2-loss Support Vector Machine & (Fan, et al., 2008) \\
\hline PAM & Nearest shrunken centroid classifier & (Tibshirani, et al., 2002) \\
\hline Constant & Majority voting classifier & - \\
\hline
\end{tabular}

\section{Results}

\subsection{Signaling pathway activity leads to improved prognosis for breast tumor samples}

The performance of using different types of profiles (Table 2) as predictive features to classify survival outcome for breast cancer patients is shown in Figure 1 Under either criterion of AUROC to evaluate the classification performance, we observe that the activity values of signaling circuits, denoted by path.vals, yield the best performance overall. In particular, they outperform the profiles based solely on gene expression values, denoted by path.genes.vals, other.genes.vals and genes.vals. In other words, we are able to integrate the expression values of path-genes into the a priori knowledge of cell signaling to obtain pathway-level features that achieve improved prognosis. Interestingly, these pathway-level features relate to biological processes and cellular functions per se. Although the pathway-level features are derived from the expression of path-genes and thus agnostic to the expression of other-genes, the inclusion of other-genes to the signaling circuits activity values, denoted by eff.and.other.genes.vals and path.and.other.genes.vals profiles, does not significantly improve the performance (no significant differences after applying a two-sided t-test comparing differences between the cross-validation AUROC scores obtained by each pair of profiles, and adjusted for multiple testing (Benjamini and Hochberg, 1995), see Table 4). 


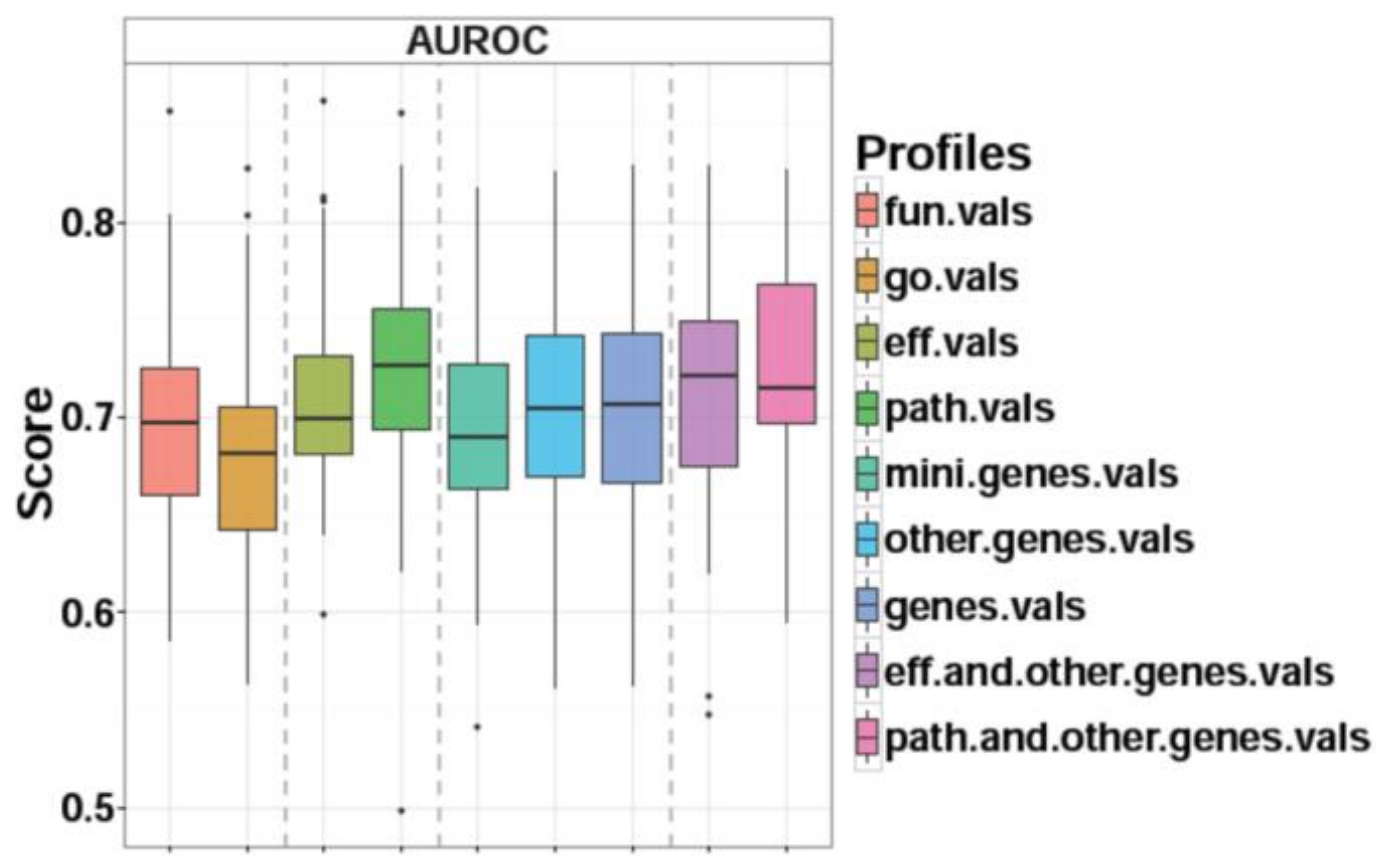

Fig. 1. The AUROC performance of using different types of profiles as predictive features to classify survival outcome for breast cancer patients. Boxplot represents the variance of the performance on 50 cross-validation splits. Dotted vertical lines separate profiles by the underlying analysis levels.

When comparing the prognostic power between pathway-level and gene-level profiles, we have also derived cellular function activity profiles, denoted by fun.vals and go.vals (Table 2), and observed that the performance of these profiles are slightly worse than other pathway-level profiles (Fig. 1). This is probably due to the excessively simplistic procedure that basically averages the signaling activity values of effector circuits ending at proteins with the same annotated keywords according to Uniprot or GO (Hidalgo, et al., 2017), annotations that can be incomplete and ambiguous to some extent.

Table 4. FDR-adjusted p-values comparing the corresponding classification scores of feature $\mathrm{s}$ in columns versus features in files over 50 cross-validation splits. See Figure 1 for the performance values of each feature. Significant values are in boldface and marked with an asterisk.

\begin{tabular}{|c|c|c|c|c|c|c|c|c|}
\hline & 1 & 2 & 3 & 4 & 5 & 6 & 7 & 8 \\
\hline 2 go.vals & & $0.0024 *$ & $0.0004 *$ & 0.1557 & $0.0120 *$ & 0.0119* & $0.0046^{*}$ & $<0.0001 *$ \\
\hline 3 eff.vals & & & 0.0815 & $0.0255^{*}$ & 0.8743 & 0.8743 & 0.6422 & $0.0235^{*}$ \\
\hline 4 path.vals & & & & $0.0046 *$ & 0.1408 & 0.1394 & 0.3702 & 0.6422 \\
\hline 6 other.genes & & & & & & 0.9483 & 0.2167 & $0.0039 *$ \\
\hline 7 genes & & & & & & & 0.2 & $0.0032 *$ \\
\hline 8 eff.and.other.genes & & & & & & & & $0.0473 *$ \\
\hline
\end{tabular}

Table 5 summarizes the best-performing classifiers for each type of prognostic profile in the sense that they are most frequently selected by internal cross-validation. Notably, it evidences that Support Vector Machines with various kernels are recurrently selected as the competent classifier in breast cancer prognosis that suits well for both gene-level and pathway-level features.

Table 5. Top two most frequently selected classifiers by internal cross-validation for each type of prognostic profile in classifying breast cancer prognosis evaluated by AUROC. 
bioRxiv preprint doi: https://doi.org/10.1101/132357; this version posted April 29, 2017. The copyright holder for this preprint (which was not certified by peer review) is the author/funder, who has granted bioRxiv a license to display the preprint in perpetuity. It is made available under aCC-BY 4.0 International license.

\begin{tabular}{lllll}
\hline Profile alias & mean & SD & Classifier 1 & Classifier 2 \\
\hline fun.vals & 0.6962 & 0.05438 & RadialSVM & GBM \\
go.vals & 0.6807 & 0.06095 & RadialSVM & LinearSVM \\
eff.vals & 0.7087 & 0.05099 & RadialSVM & LinearSVM \\
path.vals & 0.7211 & 0.06316 & RadialSVM & LinearSVM \\
path.genes.vals & 0.6938 & 0.05636 & RadialSVM & LinearSVM \\
other.genes.vals & 0.7075 & 0.05254 & LinearSVM & RadialSVM \\
genes.vals & 0.7075 & 0.05272 & LinearSVM & RadialSVM \\
eff.and.other.genes.vals & 0.7127 & 0.05838 & LinearSVM & RadialSVM \\
path.and.other.genes.vals & 0.7246 & 0.05359 & LinearSVM & RadialSVM \\
\hline
\end{tabular}

3.2 Signaling circuits selected as features relevant for cancer prognostic account for cancer hallmarks

From the clinical standpoint of cancer prognosis, we are interested in identifying a small set of biomarkers that can guide decision making in cancer prognosis. As our analysis is made at the level of pathways, we would like to detect a few signaling circuits whose activity, and thus the underlying cell functionality, has a significant impact on discriminating the prognosis classes of cancer patients. We opted for the Random Forest classifier to perform this analysis, since it simultaneously predicts the survival outcome of tumor samples and scores the importance of each feature that is ultimately used in the prediction. We focus on the feature importance measure returned by fitting a Random Forest which accounts for the mean decrease in classification performance if we randomly permute the data of the corresponding feature. Table 6 lists the five top-scored signaling circuits by fitting Random Forests with the profiles of circuit activities (denoted by path.vals). The role played by each signaling circuit in cancer progression can be inferred from the underlying cellular functions (taken from GO annotations) triggered by the last (effector) protein on the circuit. Thus, the first circuit, belonging to the HIF-1 signaling pathway, starts with the TLR4 receptor, which is known to be related to progression of several cancers (breast, ovarian, prostate and head and neck) via Lipopolysaccharide Stimulation (Yang, et al., 2014) and ends in the EDN1 effector, an hypoxia-inducible factor that mediates cancer progression (Semenza, 2012). Another relevant circuit belongs to the NF-kappa B signaling pathway and has the ILIB protein as receptor and the $C X C L 2$ as effector. Polymorphisms in the receptor have been linked to several cancers in different populations (El-Omar, et al., 2000; Lu, et al., 2005) and it has been demonstrated the role of CXCL2 in tumor growth and angiogenesis (Keane, et al., 2004). Similarly, polymorphisms in the LEP protein, the receptor of another circuit in the Adipocytokine signaling pathway, have been linked to cancer (Cleveland, et al., 2010), and its effector, the tyrosine phosphatase Shp2 (PTPN11), contributes to the pathogenesis of many cancers and other human diseases (Chan, et al., 2008). The Cell cycle signaling pathway contains another relevant circuit whose receptor TTK transmits the signal until the cohesin complex. This four proteins complex is essential for chromosome segregation and DNA repair and mutations in its component genes have recently been identified in several types of tumors (Losada, 2014). Finally, the fifth most relevant circuit, belonging to the Tight junction pathway, contains the AKT3 serine/threonine kinase with a known role in tumorigenesis (Testa and Bellacosa, 2001), is signaled by the receptor ACTN4, a protein which has been related to cell invasion and metastasis (Honda, 2015). Supplementary Table 2 shows an expanded list of top-scored 50 circuits.

Table 6. Top five circuits with the highest feature importance measure by fitting Random Forests with path.vals in classifying breast cancer prognosis.

\begin{tabular}{llll}
\hline Pathway name & $\begin{array}{l}\text { Receptor } \\
\text { Gene(s) }\end{array}$ & $\begin{array}{l}\text { Effector } \\
\text { Gene(s) }\end{array}$ & Effector protein function \\
\hline HIF-1 & GLR4 & EDN1 & Inflammatory response and angiogenesis \\
NF-kappa B & $I L 1 B$ & $C X C L 2$ & Protein phosphatase
\end{tabular}


bioRxiv preprint doi: https://doi.org/10.1101/132357; this version posted April 29, 2017. The copyright holder for this preprint (which was not certified by peer review) is the author/funder, who has granted bioRxiv a license to display the preprint in perpetuity. It is made available under aCC-BY 4.0 International license.

$\begin{array}{ll}\text { Cell cycle } & \text { Cohesin complex } \\ & (S M C 1 B, \quad S M C 3, \\ & S T A G 1, R A D 21)\end{array}$

Tight junction ACTN4, MAGI3 AKT3 Cell invasion and metastasis

Table 7. Top five effector circuits with the highest feature importance measure by fitting Random Forests with eff.vals in classifying breast cancer prognosis.

\begin{tabular}{|c|c|c|}
\hline Pathway name & Effector gene & Effector protein function \\
\hline AMPK & $L E P R$ & Regulation of fatty acid metabolism \\
\hline Adipocytokine & $P P A R \alpha$ & Peroxisome proliferation and fatty acid metabolism \\
\hline Pathways in cancer & IL6 & Blockage of differentiation, Anti-apoptosis \\
\hline Cell cycle & $\begin{array}{l}\text { Cohesin r complex } \\
(S M C 1 B, \quad S M C 3, \\
S T A G 1, R A D 21)\end{array}$ & Chromosome segregation and DNA repair \\
\hline Toll-like receptor & IL6 & Inflammation, Immune response, Anti-apoptosis \\
\hline
\end{tabular}

Table 7 lists the top-scored effector circuits by fitting Random Forests with the profiles of effector circuit activities (denoted by eff.vals). Although the cohesion complex effector is again selected, the effector circuit level analysis provided a slightly different perspective of relevant aspects of signaling in cancer patient survival. Thus, two effector circuits with effector proteins $L E P R$ and PPAR , from the AMPK and the Adipocytokine signaling pathways, respectively, are activators of the fatty acid metabolism. Two more effector pathways ending in the Interleukin 6 (IL6), related to inflammatory processes and immune response in the Toll-like receptor pathway, seem more likely to be involved in blocking the cell differentiation through the Pathways in cancer (KEGG id hsa05200). Actually, it has been described that IL6 blocks apoptosis in cells during the inflammatory process, keeping them alive in toxic environments, but the same process protects cells from apoptosis and chemotherapeutic drugs during neoplastic growth (Hodge, et al., 2005). Supplementary Table 3 shows an expanded list of top-scored 50 effector circuits. Beyond the top scored signaling circuits (Table 6) and effector circuits (Table 7), other relevant circuits are listed in Supplementary Tables 2 and 3. Although an exhaustive list of the consequences that processes differentially activated can have in tumorigenesis is beyond the scope of this work, it is worth noticing that cancer a hallmark such as apoptosis inhibition is represented by inhibition of signaling circuits IL6-BCL2 in the HIF-1 signaling pathway and IL10-BNIP3 in the FoxO signaling pathway (eighth and ninth in Supplementary Table 2, respectively), both containing the protein STAT3, known to mediate apoptosis inhibition in breast cancer (Gritsko, et al., 2006) (see Figure 2). The graphic representation of the complete effector circuits containing the two signaling circuits in the HIF-1 signaling pathway and the FoxO signaling pathway has been obtained with the hipathia web tool (Hidalgo, et al., 2017), using the path.genes.vals gene expression profiles (that are converted to eff.vals and path.vals profiles by the program). 


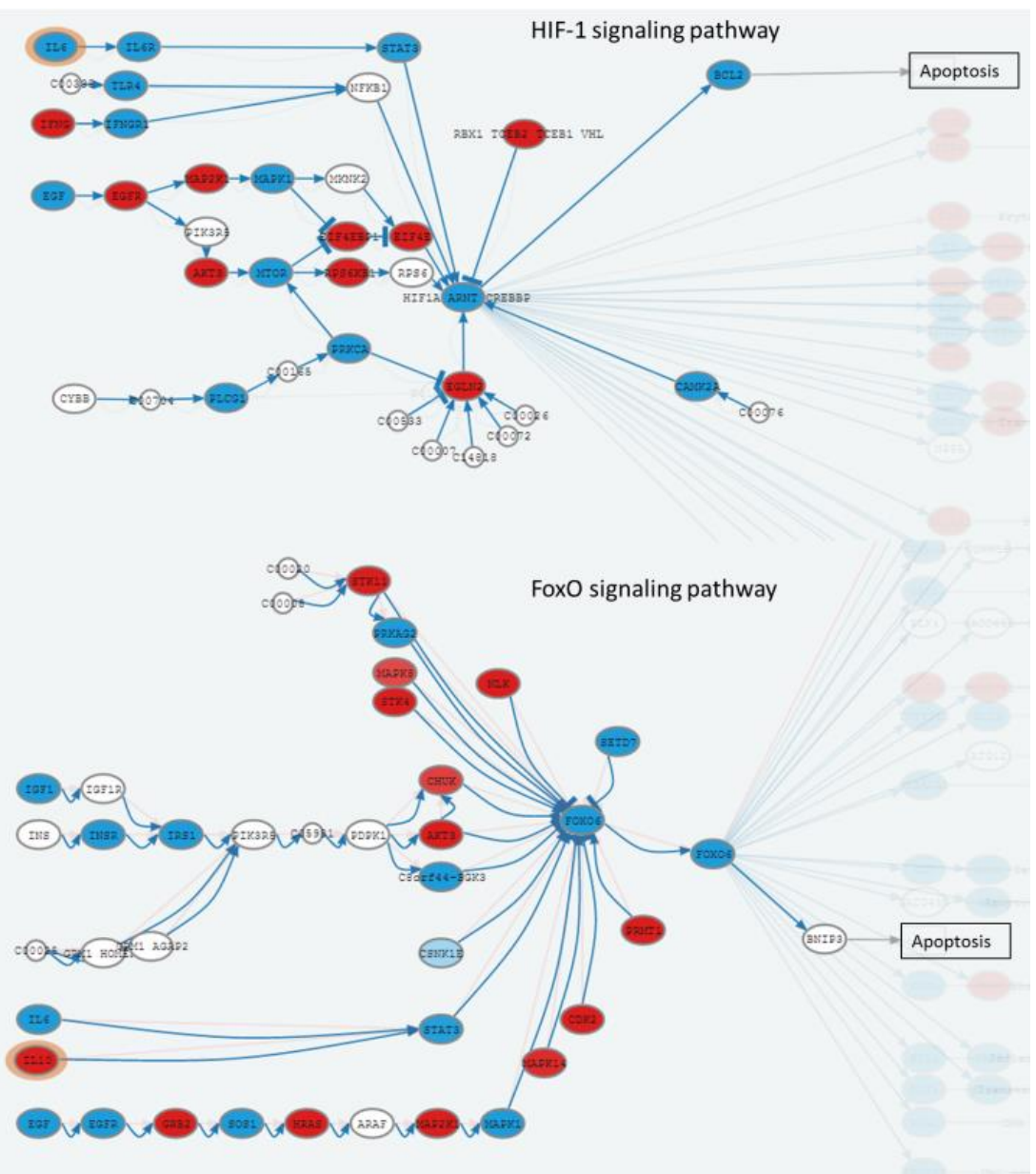

Fig. 2. Effector circuits containing Signaling circuits IL6-BCL2 in the HIF-1 signaling pathway and ILIO-BNIP3 in the FoxO signaling pathway, both highlighted in the figure. Both circuits contain the protein $S T A T 3$, known to mediate apoptosis inhibition in breast cancer.

Table 8. Top five genes unrelated to signaling ranked by importance in the classification of survival outcome by fitting Random Forests with path.and.other.genes.vals profile, along their functions as annotated in Gene Ontology.

\begin{tabular}{llll}
\hline Gene ID & Gene symbol & Gene full name & GO Function \\
\hline 6944 & VPS72 & Vacuolar protein sorting 72 homolog & DNA binding \\
150356 & CHADL & Chondroadherin like & Collagen binding \\
340273 & ABCB5 & $\begin{array}{l}\text { ATP binding cassette subfamily B } \\
\text { member 5 }\end{array}$ & $\begin{array}{l}\text { ATP binding, Efflux transmembrane transporter } \\
\text { activity }\end{array}$ \\
8543 & LMO4 & LIM domain only 4 & $\begin{array}{l}\text { Transcription factor activity, Sequence-specific } \\
\text { DNA binding } \\
4976\end{array}$ \\
OPA1 & OPA1, mitochondrial dynamin like & $\begin{array}{l}\text { GTPase activity } \\
\text { GTPase }\end{array}$
\end{tabular}

\subsection{The classification algorithm suggests additional prognostic genes that do not code for signaling proteins}

In order to find genes that could be relevant for patient survival that are not in the signal pathways, we build a profile by combining signaling circuit activity profiles and gene expression profiles corresponding 
bioRxiv preprint doi: https://doi.org/10.1101/132357; this version posted April 29, 2017. The copyright holder for this preprint (which was

to genes outside signaling pathways (the other-genes profile), denoted by path.and.other.genes.vals. A feature selection procedure in breast cancer prognosis based on such a profile can select signaling circuits along with genes unrelated to signaling, whose activity is related to patient survival. Thus, Random Forest was again used to assess feature importance when fit with the path.and.other.genes.vals profile to classify survival outcome. Table 8 lists the top 5 most important gene features (the other-genes part of the path.and.other.genes.vals composed profile). These genes are of particular interest given that they might represent relevant cancer processes not included in cell signaling. Notably, the gene $A B C B 5$ belongs to the ATP-binding cassette subfamily B which is well known to be involved in multiple drug resistance in cancer therapy (Dean, et al., 2001), probably because its functionality of efflux transmembrane transporter. It has also been reported that $A B C B 5$ could mediate cell-to-cell fusion and contribute to breast cancer chemoresistance in expressing breast tumors (Frank, et al., 2005; Frank, et al., 2003). In addition, $A B C B 5$, as a "pro-survival" gene, has been suggested to be a potential target against drug resistant breast cancer cells (Yang, et al., 2010). Besides, ABCB5 has been linked to melanoma (Wilson, et al., 2014). LMO4 encodes a LIM- domain protein that has been reported as an essential mediator of cell cycle progression in ErbB2/HER2/Neu-induced breast cancer which is characterized by poor survival due to high proliferation and metastasis rates (Matthews, et al., 2013; Montañez-Wiscovich, et al., 2009). It has been reported that $L M O 4$ interacts with the renowned tumor suppressor BRCAI and inhibits BRCAl activity (Sum, et al., 2002; Sutherland, et al., 2003). OPAl encodes a mitochondrial fusion protein which might be a target for mitochondrial apoptotic effectors (Olichon, et al., 2003), such as sorafenib (Zhao, et al., 2013). The role in cancer survival played by two most important genes according to the predictor, VPS72 and CHADL, is not as clear from the literature. It is worth mentioning that a mutation in VPS72 in cervix cancer with a high FATHMM pathogenicity score (Shihab, et al., 2015) is described in the COSMIC database (entry COSM458603). Regarding CHADL, it has been related to chondrocyte differentiation (Tillgren, et al., 2015) and extracellular matrix remodeling (Barallobre-Barreiro, et al., 2012). Therefore, both genes are potentially involved in cancer processes, which suggest that further investigation of the complete list of top-ranked other-genes could render new cancer drivers and potential therapeutic targets. An expanded list containing the top 50 most important features among the other-genes can be found in Supplementary Table 4, in which many genes with cancer-related functions can be seen. Functions for the genes have been taken from their Uniprot (UniProt_Consortium, 2015) annotations and, when absent, from GeneCards annotations (Stelzer, et al., 2016).

\subsection{Availability of data and results}

All experiments are produced with $\mathrm{R}$ and codes are available via https://github.com/YunlongJiao/hipathiaCancerPrognosis.

There is an $\mathrm{R}$ package available at https://github.com/babelomics/hipathia. Additionally, there is a web interface to the hipathia methodology that includes prediction functionalities, which is freely available at: http://hipathia.babelomics.org/.

\section{Conclusions}

In this study we have proposed a novel scheme to classify survival outcome for breast cancer patients based on mechanistic features consisting of signaling pathway activity profiles. We applied a pathway activity analysis method (Hidalgo, et al., 2017) to recode gene expression profiles into activity values of signaling circuits and demonstrated that, making use of the state-of-the-art computational tools, signaling circuit activity yields better prediction in breast cancer prognosis than gene expression. An additional advantage is that the identified pathway-level biomarkers are mechanistic signatures whose contribution to cancer progression can be readily interpreted in terms of the underlying cellular functions and biological processes.

The three feature sets path.genes.vals, eff.vals and path.vals are composed by the same genes (those present in the pathways). However, the prediction performance of the genes recoded into circuits activity values with the hipathia method (eff.vals and path.vals) clearly outperforms (see Table 4) to those of the original genes (path.genes.vals). Moreover, predictors based on circuits (eff.vals and path.vals) have similar performance (see Table 4) to predictors based on all the genes (genes.vals), which include more information than the subset of genes. It is worth noting that genes in the circuits represent only $12 \%$ of the total number of genes, but have the same predictive performance, which suggests that combining the genes into circuits provides a real added value for prediction purposes.

Although a significant improvement of the performance was not observed when the expression values of other-genes were concatenated to the activity values of signaling circuits, the analysis based on the combination of both data provides an interesting perspective regarding the interpretation of the biomarkers detected. In fact, the selected genes from the category of other-genes represent other aspects of the mechanism of the disease not explained by cell signaling. This approach allows expanding the scope of the analysis beyond the processes included in the pathways modeled. 
bioRxiv preprint doi: https://doi.org/10.1101/132357; this version posted April 29, 2017. The copyright holder for this preprint (which was

Central to our proposed scheme is the idea of promoting gene-level analysis to pathway-level analysis by obtaining patient-specific personalized profiles of signaling circuit activity. Reliable models of pathway activity (Hidalgo, et al., 2017) can be used to derive robust multigenic biomarkers, similar to the popular MammaPrint (van't Veer and Bernards, 2008), which in addition account properly for the underlying disease mechanisms or mechanisms of drug action.

\section{Funding}

This work was supported by the European Union 7th Framework Program through the Marie Curie ITN MLPM grant No 316861, by the European Research Council grant ERC-SMAC-280032, by grants BIO2014-57291-R from the Spanish Ministry of Economy and Competitiveness and "Plataforma de Recursos Biomoleculares y Bioinformáticos" PT13/0001/0007 from the ISCIII, both co-funded with European Regional Development Funds (ERDF); and EU H2020-INFRADEV-1-2015-1 ELIXIREXCELERATE (ref. 676559)

\section{References}

Amadoz, A., et al. (2015) Using activation status of signaling pathways as mechanism-based biomarkers to predict drug sensitivity, Scientific reports, 5, 18494.

Ashburner, M., et al. (2000) Gene ontology: tool for the unification of biology. The Gene Ontology Consortium, Nat Genet, 25, 25-29.

Barabasi, A.L., Gulbahce, N. and Loscalzo, J. (2011) Network medicine: a network-based approach to human disease, Nat Rev Genet, 12, 56-68.

Barabasi, A.L. and Oltvai, Z.N. (2004) Network biology: understanding the cell's functional organization, Nat Rev Genet, 5, 101-113.

Barallobre-Barreiro, J., et al. (2012) Proteomics analysis of cardiac extracellular matrix remodeling in a porcine model of ischemia-reperfusion injury, Circulation,

CIRCULATIONAHA. 111.056952.

Benjamini, Y. and Hochberg, Y. (1995) Controlling the false discovery rate: a practical and powerful approach to multiple testing, Journal of the Royal Statistical Society. Series B, 57, 289-300.

Bhardwaj, N. and Lu, H. (2005) Correlation between gene expression profiles and proteinprotein interactions within and across genomes, Bioinformatics, 21, 2730-2738.

Breiman, L. (2001) Random Forests, Machine Learning, 45, 5-32.

Cleveland, R.J., et al. (2010) Common genetic variations in the LEP and LEPR genes, obesity and breast cancer incidence and survival, Breast cancer research and treatment, 120, 745-752. Chan, G., Kalaitzidis, D. and Neel, B.G. (2008) The tyrosine phosphatase Shp2 (PTPN11) in cancer, Cancer and metastasis reviews, 27, 179-192.

Chang, C.-C. and Lin, C.-J. (2011) LIBSVM: a library for support vector machines, ACM Transactions on Intelligent Systems and Technology (TIST), 2, 27.

Dean, M., Hamon, Y. and Chimini, G. (2001) The human ATP-binding cassette (ABC) transporter superfamily, Journal of lipid research, 42, 1007-1017.

Dopazo, J. (2010) Functional profiling methods in cancer, Methods Mol Biol, 576, 363-374.

Drier, Y., Sheffer, M. and Domany, E. (2013) Pathway-based personalized analysis of cancer, Proceedings of the National Academy of Sciences, 110, 6388-6393.

Efroni, S., Schaefer, C.F. and Buetow, K.H. (2007) Identification of key processes underlying cancer phenotypes using biologic pathway analysis, PLOS ONE, 2, e425.

Ein-Dor, L., Zuk, O. and Domany, E. (2006) Thousands of samples are needed to generate a robust gene list for predicting outcome in cancer, Proc Natl Acad Sci U S A, 103, 5923-5928. El-Omar, E.M., et al. (2000) Interleukin-1 polymorphisms associated with increased risk of gastric cancer, Nature, 404, 398-402.

Fan, R.-E., et al. (2008) LIBLINEAR: A library for large linear classification, Journal of machine learning research, 9, 1871-1874.

Fey, D., et al. (2015) Signaling pathway models as biomarkers: Patient-specific simulations of JNK activity predict the survival of neuroblastoma patients, Sci Signal, 8, ra130. 
bioRxiv preprint doi: https://doi.org/10.1101/132357; this version posted April 29, 2017. The copyright holder for this preprint (which was

Frank, N.Y., et al. (2005) ABCB5-mediated doxorubicin transport and chemoresistance in human malignant melanoma, Cancer research, 65, 4320-4333.

Frank, N.Y., et al. (2003) Regulation of progenitor cell fusion by ABCB5 P-glycoprotein, a novel human ATP-binding cassette transporter, Journal of Biological Chemistry, 278, 4715647165.

Friedman, J., Hastie, T. and Tibshirani, R. (2010) Regularization paths for generalized linear models via coordinate descent, Journal of Statistical Software, $\mathbf{3 3}, 1$.

Friedman, J.H. (2001) Greedy function approximation: a gradient boosting machine, Annals of Statistics, 1189-1232.

Fryburg, D.A., et al. (2014) Systems diagnostics: anticipating the next generation of diagnostic tests based on mechanistic insight into disease, Drug Discov Today, 19, 108-112.

Gritsko, T., et al. (2006) Persistent activation of stat 3 signaling induces survivin gene expression and confers resistance to apoptosis in human breast cancer cells, Clinical Cancer Research, 12, 11-19.

Hanahan, D. and Weinberg, R.A. (2011) Hallmarks of cancer: the next generation, Cell, 144, 646-674.

Hidalgo, M.R., et al. (2017) High throughput estimation of functional cell activities reveals disease mechanisms and predicts relevant clinical outcomes, Oncotarget, 8, 5160-5178.

Hodge, D.R., Hurt, E.M. and Farrar, W.L. (2005) The role of IL-6 and STAT3 in inflammation and cancer, European journal of cancer, 41, 2502-2512.

Honda, K. (2015) The biological role of actinin-4 (ACTN4) in malignant phenotypes of cancer, Cell \& bioscience, $\mathbf{5}, 41$.

Hood, L. (2013) Systems biology and p4 medicine: past, present, and future, Rambam Maimonides medical journal, 4, e0012.

Iwamoto, T. and Pusztai, L. (2010) Predicting prognosis of breast cancer with gene signatures: are we lost in a sea of data?, Genome medicine, $2,81$.

Jacob, L., Neuvial, P. and Dudoit, S. (2012) More power via graph-structured tests for differential expression of gene networks, Ann. Appl. Stat., 6, 561-600.

Jiao, Y. and Vert, J.-P. (2016) The Kendall and Mallows kernels for permutations. HAL. pp. hal01279273.

Johnson, W.E., Li, C. and Rabinovic, A. (2007) Adjusting batch effects in microarray expression data using empirical Bayes methods, Biostatistics, 8, 118-127.

Kanehisa, M., et al. (2012) KEGG for integration and interpretation of large-scale molecular data sets, Nucleic Acids Res, 40, D109-114.

Keane, M.P., et al. (2004) Depletion of CXCR2 inhibits tumor growth and angiogenesis in a murine model of lung cancer, The Journal of Immunology, 172, 2853-2860.

$\mathrm{Li}, \mathrm{X}$., et al. (2015) Subpathway Analysis based on Signaling-Pathway Impact Analysis of Signaling Pathway, PLoS ONE, 10, e0132813.

Liaw, A. and Wiener, M. (2002) Classification and regression by randomForest, $R$ news, 2, 18 22.

Livshits, A., et al. (2015) Pathway-based personalized analysis of breast cancer expression data, Molecular oncology, 9, 1471-1483.

Losada, A. (2014) Cohesin in cancer: chromosome segregation and beyond, Nature Reviews Cancer, 14, 389-393.

Lu, W., et al. (2005) Genetic polymorphisms of interleukin (IL)-1B, IL-1RN, IL-8, IL-10 and tumor necrosis factor $\alpha$ and risk of gastric cancer in a Chinese population, Carcinogenesis, 26, 631-636.

Martini, P., et al. (2013) Along signal paths: an empirical gene set approach exploiting pathway topology, Nucleic Acids Res, 41, e19.

Matthews, J.M., et al. (2013) LIM-domain-only proteins in cancer, Nature Reviews Cancer, 13, 111-122.

Montaner, D., et al. (2009) Gene set internal coherence in the context of functional profiling, BMC Genomics, 10, 197.

Montañez-Wiscovich, M., et al. (2009) LMO4 is an essential mediator of ErbB2/HER2/Neuinduced breast cancer cell cycle progression, Oncogene, 28, 3608-3618. 
bioRxiv preprint doi: https://doi.org/10.1101/132357; this version posted April 29, 2017. The copyright holder for this preprint (which was

Olichon, A., et al. (2003) Loss of OPA1 perturbates the mitochondrial inner membrane structure and integrity, leading to cytochrome c release and apoptosis, Journal of Biological Chemistry, 278, 7743-7746.

Oti, M. and Brunner, H.G. (2007) The modular nature of genetic diseases, Clin Genet, 71, 1-11. Paik, S., et al. (2004) A multigene assay to predict recurrence of tamoxifen-treated, nodenegative breast cancer, New England Journal of Medicine, 351, 2817-2826.

Ripley, B.D. (2007) Pattern recognition and neural networks. Cambridge university press. Robinson, M.D. and Oshlack, A. (2010) A scaling normalization method for differential expression analysis of RNA-seq data, Genome Biol, 11, R25.

Sebastian-Leon, P., et al. (2014) Understanding disease mechanisms with models of signaling pathway activities, BMC Syst Biol, 8, 121.

Semenza, G.L. (2012) Hypoxia-inducible factors: mediators of cancer progression and targets for cancer therapy, Trends in pharmacological sciences, 33, 207-214.

Shihab, H.A., et al. (2015) An integrative approach to predicting the functional effects of noncoding and coding sequence variation, Bioinformatics, 31, 1536-1543.

Sing, T., et al. (2005) ROCR: visualizing classifier performance in R, Bioinformatics, 21, 39403941.

Sotiriou, C. and Pusztai, L. (2009) Gene-expression signatures in breast cancer, New England Journal of Medicine, 360, 790-800.

Stelzer, G., et al. (2016) The genecards suite: from gene data mining to disease genome sequence analyses, Current protocols in bioinformatics, 1.30. 31-31.30. 33.

Sum, E.Y., et al. (2002) The LIM domain protein LMO4 interacts with the cofactor CtIP and the tumor suppressor BRCA1 and inhibits BRCA1 activity, Journal of Biological Chemistry, 277, 7849-7856.

Sutherland, K.D., et al. (2003) Mutational analysis of the LMO4 gene, encoding a BRCA1interacting protein, in breast carcinomas, International journal of cancer, 107, 155-158.

Testa, J.R. and Bellacosa, A. (2001) AKT plays a central role in tumorigenesis, Proceedings of the National Academy of Sciences, 98, 10983-10985.

Tibshirani, R., et al. (2002) Diagnosis of multiple cancer types by shrunken centroids of gene expression, Proceedings of the National Academy of Sciences, 99, 6567-6572.

Tillgren, V., et al. (2015) The novel small leucine-rich protein chondroadherin-like (CHADL) is expressed in cartilage and modulates chondrocyte differentiation, Journal of Biological Chemistry, 290, 918-925.

UniProt_Consortium (2015) UniProt: a hub for protein information, Nucleic Acids Res, 43, D204-212.

van't Veer, L.J. and Bernards, R. (2008) Enabling personalized cancer medicine through analysis of gene-expression patterns, Nature, 452, 564-570.

van 't Veer, L.J., et al. (2002) Gene expression profiling predicts clinical outcome of breast cancer, Nature, 415, 530-536.

Venables, W. and Ripley, B. (2002) Modern Applied Statistics with S. Springer, New York.

Vidal, M., Cusick, M.E. and Barabasi, A.L. (2011) Interactome networks and human disease, Cell, 144, 986-998.

Wang, Y., et al. (2005) Gene-expression profiles to predict distant metastasis of lymph-nodenegative primary breast cancer, The Lancet, 365, 671-679.

Wilson, B.J., et al. (2014) ABCB5 maintains melanoma-initiating cells through a proinflammatory cytokine signaling circuit, Cancer research, 74, 4196-4207.

Yang, H., et al. (2014) Toll-like receptor 4 prompts human breast cancer cells invasiveness via lipopolysaccharide stimulation and is overexpressed in patients with lymph node metastasis, PLOS ONE, 9, e109980.

Yang, J.Y., et al. (2010) p-Glycoprotein ABCB5 and YB-1 expression plays a role in increased heterogeneity of breast cancer cells: correlations with cell fusion and doxorubicin resistance, BMC Cancer, 10, 388.

Zeileis, A., et al. (2004) kernlab-an S4 package for kernel methods in R, Journal of Statistical Software, 11, 1-20. 
bioRxiv preprint doi: https://doi.org/10.1101/132357; this version posted April 29, 2017. The copyright holder for this preprint (which was not certified by peer review) is the author/funder, who has granted bioRxiv a license to display the preprint in perpetuity. It is made available under aCC-BY 4.0 International license.

Zhao, X., et al. (2013) OPA1 downregulation is involved in sorafenib-induced apoptosis in hepatocellular carcinoma, Laboratory Investigation, 93, 8-19. 


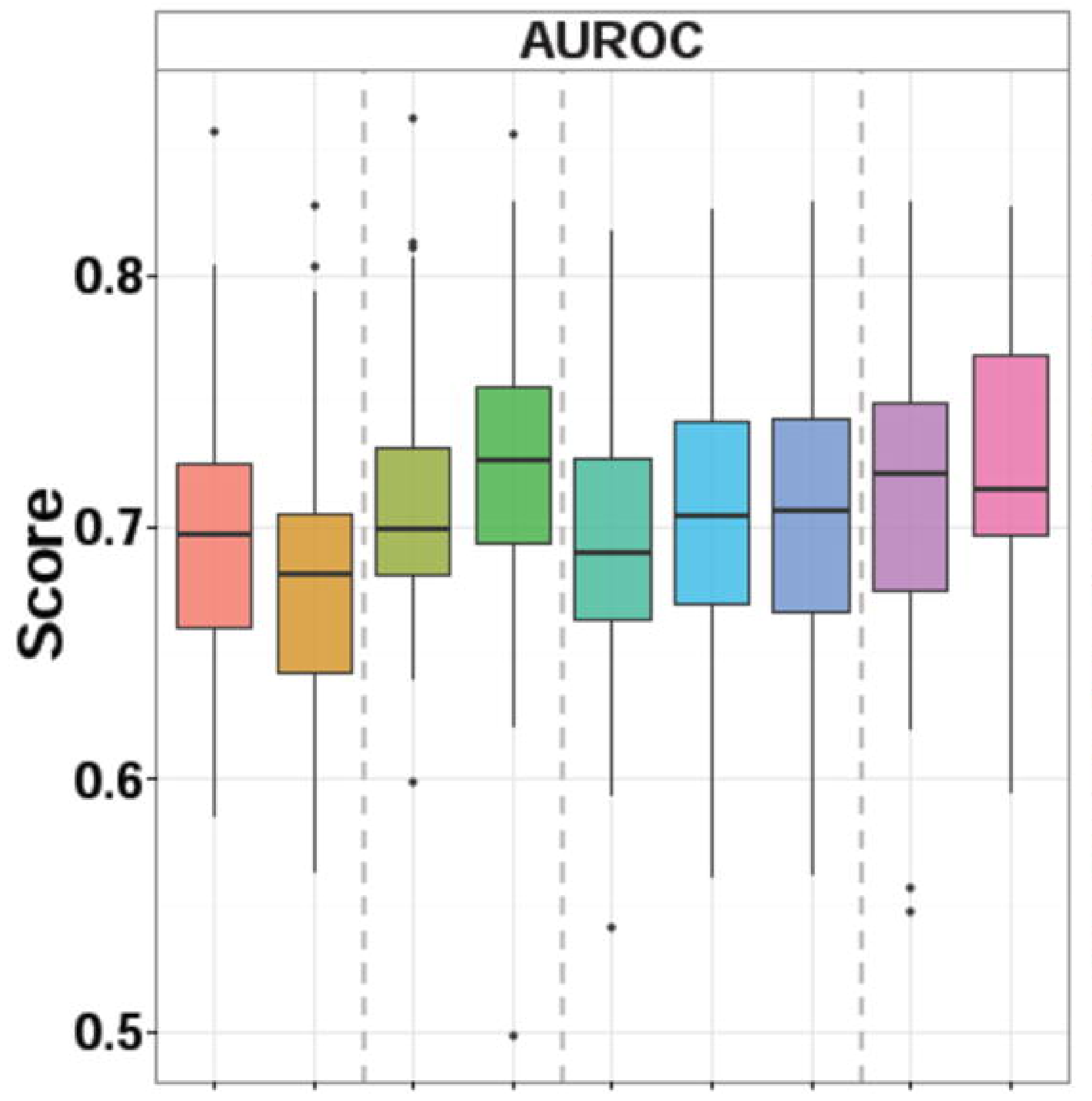

\section{Profiles}

Erfun.vals

草go.vals

Eieff.vals

主path.vals

Emini.genes.vals

草other.genes.vals

自genes.vals

草eff.and.other.genes.vals

Epath.and.other.genes.vals 


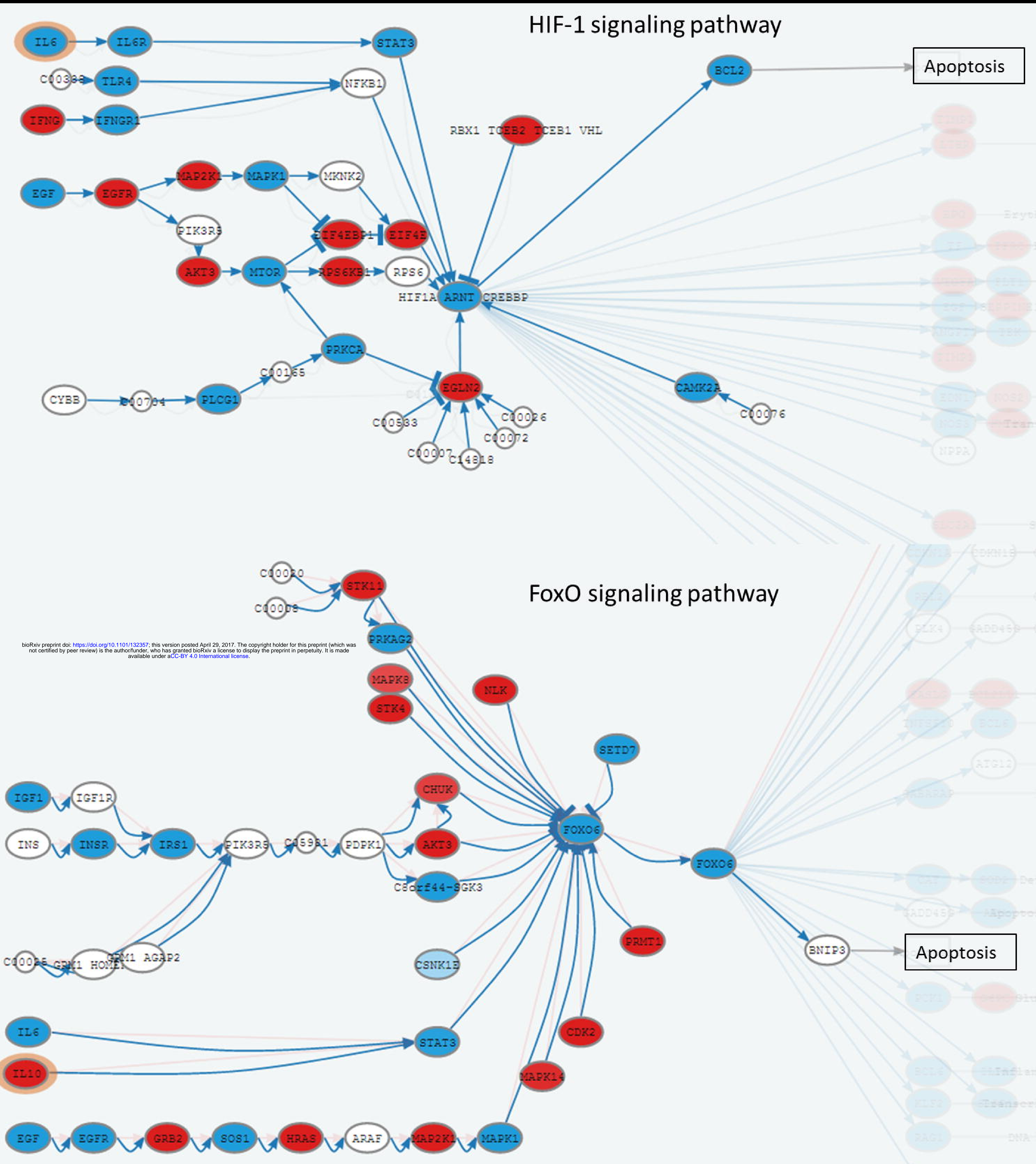

